\title{
Análisis de riesgos en la producción de vino
}

\author{
Muñoz Gustavo, Castro María, Echegaray Marcelo, Palacios Carlos y Rodriguez Rosa ${ }^{a}$ \\ Instituto de Ingeniería Química-Facultad de Ingeniería-Universidad Nacional de San Juan
}

\begin{abstract}
Resumen. La industria vitivinícola es una de las más importantes en la región de Cuyo. Analizando el proceso completo de elaboración del vino, debe tenerse en cuenta que se desarrolla en dos ámbitos totalmente diferentes: el campo y las instalaciones industriales dedicadas al procesado de la uva. Por otra parte, actualmente todas las empresas deben implantar de manera obligatoria sistemas de prevención que sean capaces de garantizar la seguridad y la salud de los trabajadores conforme lo establecido en la legislación vigente. Los accidentes laborales representan un gran daño físico, psicológico y social para el trabajador que los sufre. Además de las lesiones corporales y psicológicas originadas, la siniestralidad laboral supone un perjuicio social en el entorno del trabajador, y a su vez, un deterioro económico, ya que en la mayoría de los casos supone la ausencia o imposibilidad de ejercer la actividad productiva. Para ello, en este trabajo se realiza una identificación de los riesgos inaceptables, los cuales requieren la aplicación de medidas de reducción. En la práctica, estas medidas disminuyen la frecuencia y/o la severidad de un evento peligroso. Posteriormente se proponen diferentes medidas de prevención las cuales son de tipo, de control de procesos y medidas organizativas.
\end{abstract}

\section{Introducción}

La vitivinicultura en nuestro país, juega un importante papel que va más allá de lo estrictamente económico, pues se trata de un sector que cumple una importante función social como es la fijación de la población en el medio rural.

La producción de uva nacional, en el año 2013, fue de 2871.7 tn, destinándose a vinificación 2786.1 tn. La producción total de vinos en este año en Argentina, fue de $14983356 \mathrm{hl}$, siendo el mayor productor Mendoza, con 11775478 hl, seguido por San Juan con 2322806 hl. Por otra parte, se produjeron $21592845 \mathrm{hl}$ de mosto, siendo Mendoza el mayor productor [1].

De estos datos, se deduce que la industria vitivinícola es una de las más importantes de la región Cuyo. Este sector puede dividirse en dos tipos de actividad: por una parte, la agraria o viticultura $y$, por otra, la industrial o vinicultura.

Las bodegas están marcadas por una elevada temporalidad de los trabajadores. En la época de vendimia es cuando se concentra el mayor número de trabajadores, ya que la contratación responde a las necesidades del cultivo de la vid y a las condiciones especiales que se precisan para la elaboración del vino [2].

Considerando la totalidad del sector vitivinícola, se puede mencionar que existen riesgos asociados al cultivo de la vid y, por otro, los riesgos asociados al proceso de elaboración del vino, que engloba desde la recepción de la uva en bodega hasta la expedición del producto terminado.

Debido a la necesidad del estudio de la tipología y causas que provocan los accidentes de trabajo en la industria vinícola, en el presente trabajo se identifican los diferentes riesgos que se presentan en

\footnotetext{
${ }^{\text {a }}$ Autor de correspondencia: rrodri@unsj.edu.ar
}

cada etapa de producción, para proporcionar una serie de recomendaciones a las empresas del sector que permitan su eficaz prevención.

Actualmente todas las empresas deben implantar de manera obligatoria sistemas de prevención que sean capaces de garantizar la seguridad y la salud de los trabajadores conforme lo establecido en la Ley Nacional de Seguridad e Higiene en el trabajo, No19587 y su Decreto Reglamentario, $\mathrm{N}^{\circ} 351 / 79$. Así, ésta aporta una nueva visión de la seguridad laboral, permitiendo que la actividad preventiva quede completamente integrada en el sistema general de gestión de la empresa [3].

\section{Identificación de riesgos potenciales}

Existe una relación entre los accidentes laborales y la temporalidad, la contratación mediante empresas de trabajo temporal, la existencia de turnos y nocturnidad, la antigüedad de la empresa y los sistemas de prevención contratados por las mismas.

Los accidentes laborales representan un gran daño físico, psicológico y social para el trabajador que los sufre. Además de las lesiones corporales y psicológicas originadas, la siniestralidad laboral supone un perjuicio social en el entorno del trabajador, y a su vez, un deterioro económico, ya que en la mayoría de los casos supone la ausencia o imposibilidad de ejercer la actividad productiva [4].

La identificación y valoración de los riesgos existentes en cada proceso de la vitivinicultura son fundamentales para, posteriormente, establecer las medidas preventivas necesarias con el fin de minimizar las amenazas que estas generan en la integridad física, psíquica y social del trabajador.

This is an Open Access article distributed under the terms of the Creative Commons Attribution License 4.0, which permits unrestricted use, distribution, and reproduction in any medium, provided the original work is properly cited. 


\subsection{Riesgos potenciales asociados a la cosecha}

Los riesgos de operación de maquinaria agrícola se acentúan de acuerdo a la situación geográfica o del terreno. Respecto de la situación geográfica, se destaca especialmente la incorporación de plantaciones en laderas de cerro, en que se ha agregado un riesgo adicional al trabajo de la maquinaria en esas condiciones [7-9].

\subsubsection{Riesgos asociados al uso del tractor}

Los principales riesgos asociados al uso del tractor son los riesgos de vuelco, de atrapamiento y de caídas de personas desde tractor.

La causa básica del accidente por volcamiento, obedece principalmente a las características topográficas del terreno, a fallas de operación en las que se incluyen aspectos de sobrepeso y mala estiba de la carga y las condiciones mecánicas de tractores y equipos. Malos acoplamientos entre tractor y equipos de transporte de carga, falta experiencia o conocimientos del operador.

Los accidentes que suceden en los tractores que transmiten movimientos o fuerzas, a equipos acoplados a través de la toma de fuerza y ejes de carga, se deben a la pérdida de protecciones de la línea de transmisión, que se mantiene funcionando y no se ha desacoplado al momento de enganchar a la barra de tiro de un equipo o por realizar revisiones estando este en movimiento. También en poleas y engranajes se presentan atrapamientos, en muchos casos al momento de resbalar o caer sobre éstas.

Es frecuente en los trabajos agrícolas especialmente de cosechas, que el carro de arrastre o remolque utilizado en el transporte de cargas, representa un riesgo de caídas de personas que suben al carro a descargar o bien acomodar la carga sobre los mismos y también por ser utilizados como elementos de transporte al interior de los predios o huertos. En estas condiciones se producen caídas, en el momento que el carro es movido por el tractor, sin previa verificación de la existencia de personas sobre ellos. Estos carros, en general, son utilizados para trasladar personal, sin cumplir con las condiciones de seguridad para usarlos para transporte de personas.

\subsubsection{Riesgos asociados al uso productos fitosanitarios y abonos}

Los fitosanitarios están destinados a controlar la acción de plagas, malas hierbas, bacterias y otras formas de vida animal o vegetal perjudiciales para la agricultura, incluyéndose además los plaguicidas, defoliantes, desecantes y las sustancias reguladoras del crecimiento vegetal o fitorreguladores.

Durante la aplicación de fitosanitarios existe una amplia gama de riesgos: intoxicación, irritación, quemaduras por inhalación, ingestión o contacto con la piel, destacándose principalmente la exposición por adsorción dérmica debido a la gran cantidad de tareas manuales que se realizan en el cultivo de la vid.

\subsubsection{Riesgos asociados al trabajo a la intemperie}

Se considera trabajo a la intemperie todo trabajo realizado en exteriores, las personas que realizan este tipo de actividades se encuentran principalmente expuestas a la exposición a picaduras de insectos y a las inclemencias del tiempo como lo son: las radiaciones UV, exposición al calor y a la lluvia.

\subsubsection{Riesgos asociados a las labores manuales}

Las tareas manuales que deben realizarse en el campo, como la propia vendimia, la poda o la instalación de espalderas son trabajos duros y los trabajadores del campo sienten los resultados, sufren lesiones y dolores en espalda, brazos y manos más que ningún otro problema de salud. Una tercera parte de las lesiones que les hacen faltar al trabajo son los trastornos musculo-esqueléticos relacionados con el trabajo, comúnmente denominados esguinces y dislocaciones, y una cuarta parte son lesiones de espalda. Estas son también las causas más comunes de incapacidad.

Los esguinces y las dislocaciones son ocasionados por el movimiento excesivo al estirarse, doblarse, levantar carga, agarrar objetos, ponerse en cuclillas o torcer las manos, los hombros o el cuerpo. En general, cualquier trabajo realizado con gran fuerza, herramientas anuales, con muchas repeticiones o en una posición antinatural es arriesgado. Incluso un movimiento que de por sí no es peligroso, como estirar el brazo para tomar un objeto, o apretar una herramienta, puede poner al trabajador a riesgo de lesionarse si se repite una y otra vez.

\subsection{Riesgos asociados a la elaboración de vino}

- Durante el proceso de elaboración se llevan a cabo diversas tareas cuyo orden y duración dependen de la técnica enológica empleada [7-9]. La Fig. 1 muestra, a modo de ejemplo el proceso productivo de vino blanco común [2].

\subsubsection{Riesgos asociados a la descarga de la vendimia}

Los riesgos que se pueden encontrar en este proceso son riesgos mecánicos, de caída de personas a distinto nivel, riesgos de aglomeraciones y atropello y de exposición al anhídrido sulfuroso.

Dentro de los riesgos de tipo mecánico se puede mencionar los derivados de la utilización de máquinas y de sus partes móviles, como pueden ser aplastamiento, cizallamiento, corte o seccionamiento, enganche, atrapamiento o empuje, impacto, perforación o punzamiento, fricción o abrasión, proyección de sólidos o fluidos.

El riesgo de caída de personas a distinto nivel está dado por el riesgo de caída en la tolva, principalmente.

Los riesgos de aglomeraciones y atropello se presentan en el muelle de descarga, donde los remolques esperan para depositar su carga en la tolva de recepción.

Por último, el riesgo asociado al uso de $\mathrm{SO}_{2}$, el cual es usado como antioxidante, antiséptico y disolvente, se basa principalmente en la capacidad de combinarse con facilidad con el agua formando primero ácido sulfuroso y posteriormente ácido sulfúrico, pudiendo penetrar en el organismo a través de tres vías: respiratoria, digestiva y dérmica. 


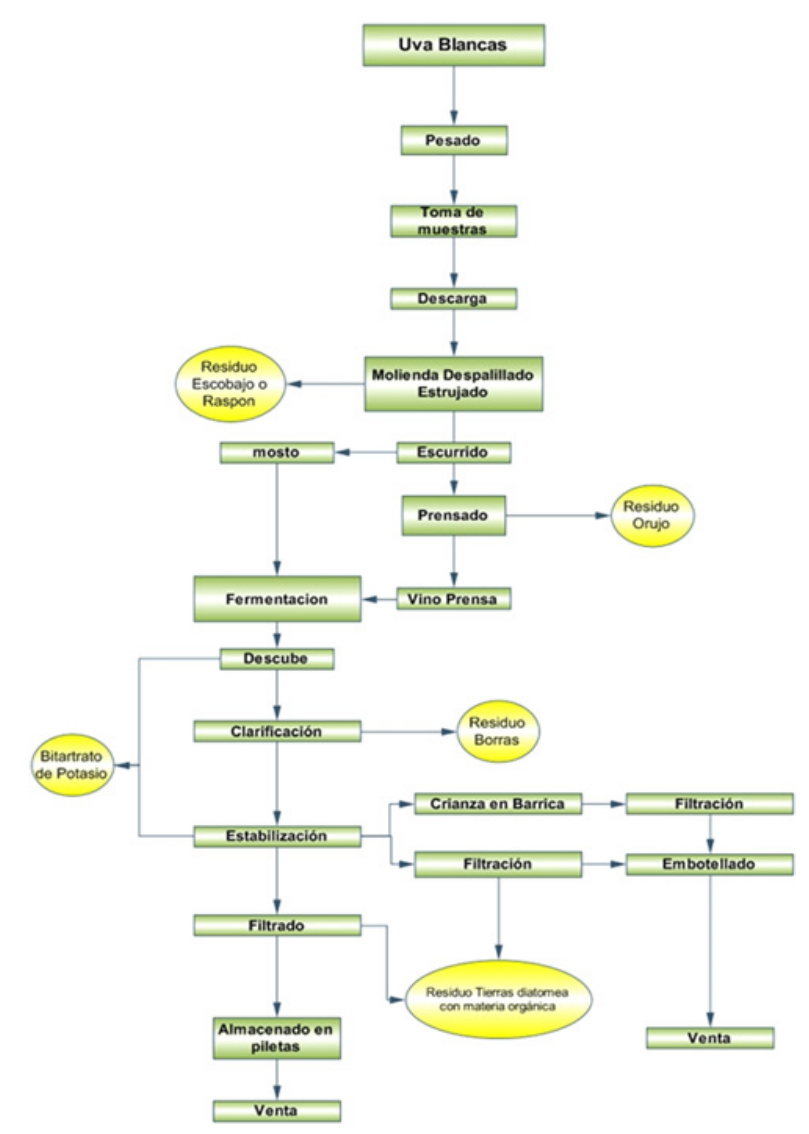

Figura 1. Esquema del proceso productivo de vino blanco.

\subsubsection{Riesgos asociados a la obtención del mosto}

Durante esta etapa se realiza el despalillado y el estrujado. Se suele disponer de un aspirador neumático a la salida de la despalilladora que retira los raspones. Para la realización del estrujado se emplean estrujadoras de rodillos, regulables en función del tamaño de la uva y de la intensidad de estrujado. También se utilizan bombas y tuberías de vendimia.

Los principales riesgos que se pueden encontrar en esta etapa son el riesgo de atrapamiento en el punto de operación, de golpes, atrapamientos y/o contactos eléctricos con las bombas y de caída a distinto nivel ya que el mosto se impulsa mediante bombas a los depósitos destinados para tal fin.

\subsubsection{Riesgos asociados a la fermentación del mosto}

Este proceso incluye la recepción, elaboración, y preparación de vinos antes del embotellado, empleándose para ello mangueras móviles y bombas de trasiego, que conducen los mostos hasta depósitos. Los mismos pueden ser autovaciantes (con fondo inclinado, para facilitar la extracción de los orujos o sólidos) o disponer de un sistema de hélices o paletas con distintos tipos de cierre.

Los riesgos que se presentan durante esta etapa son los riesgos de golpes o atrapamientos con los elementos de descube y caída a distinto nivel y de exposición a sustancias nocivas o tóxicas $\left(\mathrm{CO}_{2}\right.$ y soda cáustica).

\subsubsection{Riesgos asociados al prensado, trasiegos-crianza, clarificación y filtración}

Durante estas etapas de elaboración del vino se pueden mencionar los siguientes riesgos: de caída al mismo (debido a los derrames) y distinto nivel, de atropello por manipulación de carretillas. Para el movimiento de las barricas de una zona a otra, de disconfort por proceso húmedo, de caídas de objetos desprendidos (durante el apilado de barricas), de atrapamientos, cortes y contactos eléctricos (en el manejo de bombas y mangueras), de golpes y/o cortes (en la manipulación de las barricas), de quemaduras (durante el uso de pastillas de azufre), de exposición a sustancias tóxicas $\left(\mathrm{CO}_{2}\right.$, productos de limpieza y metabisulfito de potasio).

\subsubsection{Riesgos asociados al embotellado}

El proceso de embotellado se inicia con el lavado que comienza con la despaletizado manual o automática de las botellas. Para esta tarea suelen utilizarse herramientas cortantes, como cúter, navajas o cuchillos y plataformas móviles en altura. Luego las botellas van a la enjuagadora. A continuación se procede al llenado y encorchado. Finalmente, se lleva a cabo el etiquetado y encapsulado. El operario atiende el abastecimiento de cápsulas, etiquetas y códigos de barras. Las cajas o bolsas de estos materiales son manipuladas por el trabajador y suelen estar depositadas a pie de línea. Al finalizar la producción se limpia la máquina de restos de cola, utilizándose agua caliente y trapos, y se ordena el puesto de trabajo.

Los riesgos que se presentan durante esta etapa son los riesgo de caídas a distinto nivel (presente al desplazarse por encima de los pallets o en operaciones de despaletización de botellas), de golpes y cortes, de caídas de objeto por manipulación y/o derrumbe, de proyección de fragmentos, de atrapamientos, de sobreesfuerzo, de contactos eléctricos, de exposición al ruido, derivados del uso de productos químicos (productos de limpieza, sustancias nocivas como pegamentos y resinas, durante el etiquetado, entre otros).

\subsubsection{Riesgos asociados al embalaje, paletizado, añejamiento, almacén y expedición}

Durante estas etapas de elaboración del vino se pueden mencionar los siguientes riesgos: de caída de elementos en manipulación, de golpes y/o atrapamientos, de caída a distinto nivel, de sobreesfuerzos, de contactos eléctricos, de caída al mismo y distinto nivel y de atropellos.

\subsubsection{Riesgos derivados de la realización de actividades auxiliares}

Los riesgos derivados de las labores auxiliares que se darán en la bodega, como son administración, laboratorios y mantenimiento son los siguientes: riesgos de golpes y cortes con objetos (tijeras, grapadoras, taladradoras, guillotina, cajones, aristas cortantes, etc.), de caídas al mismo y distinto nivel (desde escaleras, zonas de trabajo elevadas, huecos o aberturas, etc., o debido a las características del suelo, o un mal orden y limpieza del lugar de trabajo), de contactos eléctricos, de exposición a 
ruido, de contacto con sustancias químicas, de incendio, riesgos derivados de equipos con pantallas de visualización de datos y de las condiciones del local de trabajo (temperatura, corrientes de aire, humedad, etc. ), riesgos de sobreesfuerzos, de desplome o caída de objetos, de golpes y/o cortes con objetos o herramientas, riesgos derivados del tránsito por la bodega, riesgo de proyecciones, de golpes, contactos, atrapamientos y de quemaduras por soldadura.

\section{Medidas de prevención}

Para conseguir el efecto deseado de la legislación y reglamentación en prevención de riesgos laborales, es preciso un cambio en la cultura preventiva de la sociedad que conceda mayor importancia a prevenir los riesgos derivados del trabajo y apueste firmemente por la integración de la prevención en las empresas.

La prevención de riesgos laborales es de aplicación inmediata, reduce la siniestralidad laboral y genera ahorros a los empresarios. Se establece como objetivo principal servir de ayuda a todos los trabajadores del sector vitivinícola en la tarea de crear espacios de trabajo más seguros, gestionar de una manera eficaz la actuación en prevención de riesgos laborales y reducir la siniestralidad laboral del sector. Todo ello repercutirá indiscutiblemente tanto en la mejora de las condiciones de seguridad y salud de los trabajadores, como en una mejor gestión de los recursos preventivos y productivos de la empresa.

\subsection{Medidas de prevención en la cosecha}

\subsubsection{Recomendaciones para evitar riesgos por el uso del tractor}

- Los conductores de tractores y maquinaria agrícola, deben ser capacitados y entrenados en la conducción y operación de los mismos.

- La supervisión debe implementarse como un sistema de gestión que incluya la seguridad de los trabajadores, dentro de las responsabilidades propias de los supervisores. La supervisión debe contribuir a valorar la seguridad industrial al interior de la empresa.

- Se deben implementar programas de mantenimiento preventivo tanto de los tractores como de los equipos agrícolas. Estos programas deben basarse en los manuales de operación y mantenimiento de los equipos agrícolas.

- Seleccionar tanto tractores como carros de arrastre de acuerdo al tipo de tarea.

- El operador debe mantenerse atento permanentemente a las condiciones de conducción para evitar atropellos, golpes y otros accidentes.

- La información técnica requerida para la operación y mantenimiento de tractores y maquinaria agrícola debe ser conocida por sus conductores y operadores.

- A mayor distancia entre ruedas (longitud de ejes) mayor seguridad. Por supuesto, debe ser compatible con el cultivo.

- Colocar el cerrojo de bloqueo de los pedales del freno del tractor cuando se circula a velocidades relativamente elevadas.
- Siempre se debe considerar la condición del suelo o terreno por el cual se desplaza, seleccionando previamente a la conducción, marchas lentas y seguras cuando se transita por pendientes, con o sin carro o equipo de arrastre. No se debe intentar el cambio de marcha una vez iniciado el desplazamiento. Se recomienda que las pendientes del terreno no deben ser superiores a los $18^{\circ}$ para trabajo longitudinal y no superior a $\operatorname{los} 11^{\circ}$ a $13^{\circ}(20-25 \%)$ para trabajos laterales. Todos los tractores que trabajan en condiciones de pendientes, deben disponer de barras anti vuelcos o cabinas de protección.

- No transitar en las proximidades de taludes, orillas de las zanjas u otras depresiones. Se recomienda circular a una distancia prudencial.

- Una cuesta acentuada se subirá marcha atrás y se bajará hacia adelante, de manera que el tractor siempre esté en la misma dirección y sentido.

- Se debe emplear el cinturón de seguridad sólo cuando el tractor cuenta con una estructura adecuada. Hoy en día es posible acoplar una estructura de seguridad a tractores que no cuenten con ella.

- Los carros de arrastre y tiro deben disponer adicionalmente de cadenas de seguridad acopladas entre la barras de tiro del carro y barra de tiro del tractor. Estas actúan en caso de desenganche de ambas barras desde su punto de acople.

- El tractor usado en trabajos con pendiente debe tener doble tracción en sus ruedas.

- Los tractores y equipos de arrastre o tiro, deben estar en buenas condiciones mecánicas, en especial el sistema de frenos y buen estado de sus neumáticos.

- El punto de acoplamiento entre tractor y carro de arrastre $\mathrm{u}$ otros equipos, debe estar asegurado correctamente en su punto de enganche, con pasador y chavetas. El enganche de remolques pesados debe realizarse en el punto más bajo.

- Las cargas a transportar en los carros de arrastre o remolques no deben exceder la capacidad de tiro del tractor y tener en cuenta las condiciones del terreno. La norma indica que el peso a transportar debe corresponder al esfuerzo que puede soportar el acople de ambas barras de tiro. La carga no debería superar el peso neto del tractor.

- Cuando los tractores se desplazan con pesos suspendidos sobre sus sistemas hidráulicos (arados, rastras, pulverizadores y otros), debe asegurarse una posición de traslado, respecto del sistema hidráulico. Para evitar movimientos laterales del implemento agrícola suspendido, esquivando los coletazos laterales que se producen al moverse el tractor por caminos con baches, piedras y otros.

- Por ningún motivo se pueden trasladar personas y niños en el tractor o el equipo acoplado a él. Los tractores y equipos agrícolas no disponen de ningún lugar seguro para el transporte de pasajeros.

- La primera recomendación indica que todas las transmisiones de movimiento deben tener protecciones que cubran completamente toda el área de transmisión. Estas protecciones deben cumplir estándares de resistencia y diseño para cubrir eficazmente los 
puntos de transmisión. Las protecciones deben estar completas y bien mantenidas en los puntos de protección.

- Establecer procedimientos de trabajo para el acople y desacople de equipos a líneas de transmisión de movimiento. Se debe indicar claramente la secuencia de operación, el uso de ropa ajustada y cómoda, herramientas y otros elementos de apoyo.

- Quienes conducen u operan tractores y maquinaria con transmisión de fuerza o movimiento, deben asegurarse que las protecciones están en su lugar y protegen completamente la línea de transmisión.

- Nunca realizar revisiones estando la maquinaria en movimiento en sus puntos de transmisión.

- Jamás ubicarse cerca de los puntos de transmisión de movimiento, especialmente si usa ropa de trabajo suelta.

- Si el sistema de transmisión de movimiento se detiene por razones de falla del sistema, atascamiento u otra razón, al revisar asegurarse que toda la línea está detenida o desconectada desde el motor hacia la transmisión.

- Considerar también los movimientos inconclusos del recorrido final del punto de operación. Ej: recorrido de cuchilla segadora atascada por una piedra.

- Cuando se realiza mantención de la maquinaria agrícola y se retira la protección para revisión y mantenimiento de partes móviles, éstas deben ser repuestas en su totalidad una vez terminada la labor $[5,6,10]$.

\subsubsection{Recomendaciones para evitar riesgos asociados al uso productos fitosanitarios y abonos}

Durante el almacenamiento:

- Los locales de almacenamiento deben llevar a la entrada el letrero "Almacén de productos químicos" y el símbolo de peligro. El local deberá ser seco, ventilado y su puerta de acceso deberá llevar cierre con llave. Además, deberá estar alejado de materiales fácilmente inflamables, como combustibles, escobajos, etc.

- La protección eléctrica deberá tener una protección adecuada, de modo que si se produce algún cortocircuito, la chispa no entre en contacto con el aire del almacén, ya que éste puede ser explosivo o inflamable, debido a la emanación de gases de los productos.

- Queda prohibido encender cigarrillos, instalar estufas o chimeneas en el local de almacenamiento.

- Los productos se almacenarán clasificados y separados por utilidades (insecticidas, herbicidas, abonos, etc.). Se situarán en estanterías, de modo que las zonas inferiores se ubiquen los productos menos tóxicos y en las superiores los más tóxicos.

- Los envases parcialmente usados deben cerrarse herméticamente para evitar posibles derrames y emanaciones de productos. Es preferible usar envases de tamaño adecuado a la cantidad de plaguicida a que se vaya a usar.

- Si se producen derrames de productos por caída y rotura de envases, se procederá a limpiar los lugares contaminados con elementos absorbentes que se recogerán y tratarán como residuos peligrosos.

Durante el transporte:

- Los plaguicidas deberán transportarse en envases resistentes y seguros y hay que evitar cualquier golpe o fricción que pudiera afectar el contenido. Se debe transportar separado de otros materiales.

Durante el tratamiento:

- Usar únicamente productos autorizados. Deben estar debidamente etiquetados y disponer de su hoja de seguridad. No comprar productos a granel ya que supone un riesgo de accidentes o errores y consecuentemente, peligro para la salud. Se deben seguir las instrucciones de formulación indicadas en la etiqueta del producto.

- Usar los productos menos tóxicos y más específicos para la plaga a tratar, para lo cual es necesario el asesoramiento técnico. Respetar, en primer lugar, los plazos de seguridad de aplicación de los productos.

- La aplicación del plaguicida debe realizarse de espaldas al viento y en sentido secuencial hacia él, de modo que se eviten nubes tóxicas que se forman y las partes del terreno tratadas.

- No soplar ni aspirar boquillas obstruidas, tampoco aspirar a través de tubos de goma para trasvasar los productos de envases.

- Las máquinas usadas para el tratamiento se emplearán sólo para este fin.

- Durante los tratamientos no se deberá comer, beber o fumar. En cualquier caso, lavarse las manos y cara con abundante agua y jabón antes de hacerlo.

- Emplear los equipos de protección individual adecuados: guantes de nitrilo o neopreno (los guantes de cuero no son válidos), buzo de protección, botas, protección para los ojos y protección respiratoria (la mascarilla de papel filtrante es insuficiente). Cómo mínimo se recomienda emplear un filtro A2-B2-P3; consultar en cualquier caso con el vendedor del producto.

- Si es posible, usar pulverizadores de boquilla larga, reduciendo bastante la exposición del trabajador al plaguicida.

- Si los trabajadores tienen heridas o rozaduras en las manos, no deben intervenir en la preparación y/o aplicación de plaguicidas.

Después del tratamiento:

- Lavar la ropa usada y revisar y cambiar, si es preciso, loe elementos de protección personal, a fin de mantenerlo en perfecto estado.

- Si después del tratamiento quedan restos de productos, éstos se guardarán en recipientes herméticos debidamente etiquetados con el nombre de producto, su composición y la palabra tóxico perfectamente visible.

- Los envases serán considerados como residuos peligrosos.

- Se deberán cumplir los plazos de seguridad antes de la cosecha o de la entrada en la zona tratada $[5,6,10]$. 


\subsubsection{Recomendaciones para evitar riesgos asociados al trabajo a la intemperie}

- Disponer siempre de agua a mano del personal y consumir frecuentemente. Es importante realizar descansos para su aclimatación.

- Llevar ropa adecuada, utilizar gorras, sombreros y cremas de protección en días de mucho sol. Evitar llevar prendas de color negro, usando preferentemente el color blanco.

- En cuanto a la exposición a picaduras de insectos, es necesario tener a mano antihistamínicos adecuados. Evitar que personas con hipersensibilidad a picaduras efectúen labores de vendimia.

- Dotar a los trabajadores expuestos con camisas de manga larga y pantalones, la idea es cubrir la mayor cantidad de piel del sol y de las picaduras.

- Evitar el trabajo a la intemperie en caso de lluvia.

- Dotar de ropa abrigadora y que a la vez no permita la acumulación de sudor corporal.

- Ingerir líquidos calientes para recuperar la temperatura perdida en caso de condiciones de frío o lluvia. Prestar mayor atención en proteger las extremidades ya que están más expuestos al frío que el resto del cuerpo.

- Usar varias capas de ropa ya que de esta manera se aísla de mejor manera el frío y en caso de sentir calor se pueden quitar las capas que se estimen conveniente. Usar botas impermeables y aislantes para evitar mojarse $[5,6,10]$.

\subsubsection{Recomendaciones para evitar riesgos asociados a labores manuales}

- Se deberá emplear el número de trabajadores necesario para la manipulación de alambres, listones de madera, etc., en la instalación de las espalderas.

- Tomar el racimo por la base, nunca por el rabo debido al riesgo de corte originado por tijera o navaja.

- Es importante utilizar guantes frente al riesgo mecánico que no hagan perder la destreza.

- No saltar entre los caballones y acequias.

- Utilizar calzado apropiado.

- Cuando las herramientas requieren fuerza, el tamaño de los mangos deberá permitir al trabajador tomar alrededor del mango de forma que el dedo índice y pulgar estén superpuestos en $0.375^{\prime \prime}$. El diámetro del mango debe oscilar entre $1.375^{\prime \prime}$ para los trabajadores de manos pequeñas y $2.125^{\prime \prime}$ para los trabajadores de manos grandes, con un promedio de $1.75^{\prime \prime}$.

- Los mangos deben estar cubiertos con un material antideslizante liso (plástico o caucho). Las herramientas de mango doble (como las tijeras de podar o los alicates) deben tener una longitud de mango de al menos $4^{\prime \prime}$ y preferiblemente de $5^{\prime \prime}$. Deben tener un dispositivo de muelle o resorte para mantener la herramienta en posición abierta y mangos que sean casi rectos sin ranuras para los dedos.

- Tener especial cuidado durante las labores de carga y descarga de cestos de vendimia para disminuir el riesgo de caídas.
- Mantener la carga que se levanta a un nivel entre manos y el nivel de los hombros. Evite levantar carga desde el suelo o por encima del nivel de los hombros.

- Usar recipientes con agarraderas o asas.

- Reconfigurar las cargas a fin de que puedan levantarse cerca del cuerpo.

- Proporcionar carretillas, carretillas hidráulicas, o carritos para objetos que sea necesario transportar por más de unos cuantos pies.

- Mantener el peso de la carga por debajo de las $22.68 \mathrm{~kg}$. Realizar pausas periódicas durante la vendimia $[5,6,10]$.

\subsection{Medidas de prevención en la producción del vino}

\subsubsection{Recomendaciones para evitar riesgos asociados a la descarga de la vendimia}

Riesgos mecánicos

- Trabajar siempre con las protecciones originales de las máquinas, sobretodo de todos los elementos móviles de ésta.

- Prestar máxima atención en tareas de limpieza, mantenimiento y/o eliminación del escobajo, especialmente si se realiza de forma manual.

- Colocar barandillas protectoras que limiten el acceso a la maquinaria y protejan al trabajador.

Riesgos de caída a distinto nivel

- Sólo personal de la bodega cualificado debe trabajar en esta zona.

- Usar plataformas elevadoras que es un sistema formado por dos rampas en línea, una de ellas para la colocación del remolque y otro armazón que esconde la tolva en su interior.

- Las rampas deben estar limpias y secas.

- Para impedir la caída de los trabajadores al interior de la tolva se deberá proteger a la misma mediante barandillas de seguridad a su alrededor. En su superficie, dotarla de una serie de perfiles metálicos horizontales, formando una parrilla, y ubicados a una distancia inferior a dos metros sobre el nivel de los dispositivos evacuadores del fondo.

- Las pasarelas deben tener piso antideslizante.

- Es fundamental que la plataforma y el tornillo sinfín tengan un dispositivo de activación dentro de una sala de control y fuera desde la misma donde el trabajador puede disponer de una amplia visión de la tolva de recepción, controlando en todo momento el proceso.

- Usar cinturones o arneses de seguridad con sistemas de sujeción para los operarios que efectúen tareas de descarga.

- El tornillo sinfín, deberá disponer de sistemas de parada de emergencia colocados en lugar visible y fácilmente accesible.

- Los toldos de los remolques deben ser retirados, pues reducen la visibilidad y pueden ocasionar accidentes graves si se sueltan de forma inesperada sobre la tolva.

- Al realizar cualquier tipo de intervención o tarea en el interior de la tolva, desconectar la máquina y retirar la llave de seguridad. 
- Elaborar protocolos y procedimientos seguros de descarga que sean conocidos por los trabajadores que realizan estas tareas.

Riesgos de aglomeraciones y atropellos

- Para la descarga de cestas de forma cómoda y segura, disponer de escaleras con peldaños y rejilla metálica.

- Respetar el turno de llegada en la línea de descarga situarse de forma segura sin producir entorpecimientos del tráfico rodado y no abandonar la cabina del vehículo sin comprobar antes que las condiciones de seguridad de la zona son las más adecuadas.

- Colocar señalizaciones y advertencias sobre este riesgo en la zona de muelle de descarga.

Riesgo de exposición al $\mathrm{SO}_{2}$

- Realizar un uso responsable del $\mathrm{SO}_{2}$ ya que un exceso de dosis no supone mayor eficacia, poniendo especial atención a las fugas.

- Se deberá prevenir los efectos de posibles salpicaduras.

- El envase debe estar siempre etiquetado y la ficha de seguridad colgada del mismo. Revisar periódicamente las botellas, válvulas, dispositivos de dosificación o inyección, etc., se debe guardar separada de las materias primas, en un lugar bien ventilado. $\mathrm{Su}$ manipulación se debe realizar únicamente por personas formadas y con experiencia.

- Si se utiliza este producto en disolución, deben usarse siempre protectores de las vías respiratorias, como mascarillas con filtros químicos.

- Evitar el riesgo de contacto con las manos usando guantes impermeables para protección contra productos químicos orgánicos.

- Cuando se produzcan concentraciones elevadas de producto en el ambiente de trabajo que pudieran ocasionar irritaciones en los ojos de los trabajadores, se aconseja el uso de protección ocular y/o gafas herméticas.

\subsubsection{Recomendaciones para evitar riesgos asociados a la obtención del mosto}

Riesgo de atrapamiento

- No retirar las protecciones originales de la estrujadora.

- No reparar ni manipular la máquina cuando ésta se encuentre en marcha.

- Señalizar en todo momento el peligro y las prohibiciones anteriormente descritas.

Riesgos de caída a distinto nivel

- Para acceder a la parte superior de los depósitos es importante que la colocación de las escaleras sea la adecuada. Estas deben estar adecuadamente protegidas. Los pasillos y pasarelas elevadas deben disponer de suelo antideslizante y barandillas con barra intermedia y rodapié.

- Es muy importante el orden y limpieza de estas superficies para evitar tanto la caída de los operarios como de objetos que pueden lesionar a personas que trabajan en niveles inferiores.

Riesgo de golpes, atrapamientos y/o contactos eléctricos con las bombas
- Deben estar dotadas de protección frente a atrapamientos protegiéndose sus conexiones y circuitos eléctricos frente a la humedad $[10,11]$.

\subsubsection{Recomendaciones para evitar riesgos asociados a la fermentación del mosto}

- Colocar, en los elementos de descube, un enrejillado similar a los de la tolva, no debiendo el operario colocarse ni apoyarse en los bordes superiores de los equipos de descube, aunque posean de dicha protección.

- Revisar la estabilidad de la escalera que se utiliza para las operaciones de acceso a depósitos o fosos, utilizando arneses y sistemas mecánicos de elevación y realizándose siempre con equipos de trabajo y procedimientos adecuados: un operario deberá estar en el exterior y permaneciendo atento. Si el trabajador del interior aprecia cualquier síntoma de asfixia deberá tirar de la cuerda para ser socorrido por el trabajador que vigila pudiendo ser necesario que deba hacer uso del equipo de salvamento con botella de oxígeno, instalada a tal efecto en esta zona.

- El diseño de la bodega y su construcción deberá contemplar que no se deba acceder al interior de los depósitos para efectuar las tareas de elaboración. También se evitará la construcción de espacios subterráneos, como fosos destinados a la ubicación de despalilladoras y estrujadoras, etc. así como la construcción de depósitos enterrados que sólo dispongan de una o dos aberturas superiores.

- Se aconseja que el depósito sea aireado antes de entrar en él, manteniéndose en marcha el ventilador hasta que finalice el descube. Además, las lámparas utilizadas para iluminar el depósito deberán disponer de mango de seguridad, grado de protección adecuado a la humedad y estarán alimentadas con tensión de seguridad. El trabajador será relevado periódicamente. Estas mismas precauciones serán observadas en todas las tareas en las que se requiere el acceso dentro de los depósitos.

- Respecto al uso de los equipos de detección y ventilación, se recomienda en primer lugar el empleo de material detector específico con doble alarma (sonora y visual) y sonda a distancia cuyo sensor debe ser reemplazado asiduamente. En segundo lugar, se aconsejan equipos de ventilación, siendo los más eficaces los que introducen aire en el fondo del depósito mediante un ventilador centrífugo o radial, debiendo tener las dimensiones correctas. También es importante que haya una adecuada ventilación forzada en las naves de fermentación y en todas aquellas zonas bajas donde puede acumularse $\mathrm{CO}_{2}$.

- Si se usa soda cáustica, que el personal responsable de manejar este producto conozca los riesgos de su exposición.

- La aplicación de pinturas de resina epoxídica en cubas y depósitos aseguran la remoción menos dificultosa del tártaro y de las partículas sólidas que quedan pegadas en las paredes después de la fermentación, evitando al máximo la utilización de esta sustancia. 
- En el caso de no poder evitar su empleo, se recomienda el uso de las protecciones individuales pertinentes, como mascarillas y guantes [10-12].

\subsubsection{Recomendaciones para evitar riesgos asociados al prensado, trasiegos - crianza, clarificación y filtración}

- Usar siempre calzado de seguridad, que posea suela antideslizante y puntera reforzada.

- Las naves deben disponer de canales de evacuación del agua y deberán estar cubiertos con rejillas.

- Los posibles derrames que se produzcan durante el prensado, deben limpiarse de inmediato para evitar que las superficies sean resbaladizas.

- Nunca colocarse o apoyarse sobre la parte superior de las prensas.

- Los operarios que manipulen las carretillas elevadoras y traspaletas deben estar capacitados en cuanto a su manejo e informados de los riesgos que su uso puede producir.

- La velocidad de transporte de las carretillas deberá adecuarse a las diferentes zonas, ya que influye en la estabilidad de la misma. Todas las carretillas cumplirán con la normativa de seguridad, realizándose su mantenimiento periódico, de acuerdo con lo establecido por el fabricante. El uso del cinturón de seguridad en las carretillas elevadoras será obligatorio y se seguirá en todo momento el diagrama de cargas de la carretilla, no superándose nunca la capacidad de carga de ésta.

- Señalizar adecuadamente las vías de movimiento en la bodega.

- Cuando los traslados de mostos se realicen a depósitos es aconsejable mantenerlos cerrados al desplazarse por el piso superior y si están abiertos se deberá disponer de protección anti caída o de rejillas que permiten la aireación del vino, pero que a su vez protegen de este riesgo.

- Las barandillas se conservarán en buen estado, revisándose periódicamente. Éstas deberán tener una altura de $90 \mathrm{~cm}$, listón intermedio y rodapié de $15 \mathrm{~cm}$.

- Cuando los trasiegos se realicen a barricas y éstas se apilen, se deberán tomar las medidas oportunas para evitar caídas durante esta operación, como pueden ser el uso de arneses o no superar la altura deapilado recomendada.

- El uso de sistemas de apilado que reducen la manipulación manual de las barricas y aumentan la seguridad del proceso evitan el desplome. Dentro de estos sistemas podemos mencionar los durmientes, que se deberán revisar periódicamente para evaluar su buen estado.

- Está prohibido situarse debajo de las barricas cuando están siendo manipuladas con las pinzas en el tren de lavado. Se realizará mantenimiento periódico de las máquinas, en especial de sus elementos de seguridad, de acuerdo con las instrucciones del fabricante.

- En cualquier caso, el personal responsable de estas operaciones debe estar adecuadamente capacitado.
- Las bombas de trasiego deben tener protección frente a atrapamientos y sus conexiones y circuitos eléctricos debidamente protegidos frente a la humedad.

- En las inmediaciones del tren de lavado de barricas nunca deberá trabajarse con brazaletes, colgantes, anillos u otros elementos que puedan aumentar el riesgo de atrapamiento o enganches con elementos móviles. En caso de tener pelo largo deberá llevarse recogido. Los puños de los buzos y camisas deberán ser ajustados.

- No introducir las extremidades en zonas móviles de las maquinas cuando están en funcionamiento. Si fuera necesario manipular elementos peligrosos, se deberá desconectar la máquina de toda fuente de energía y señalizar.

- La automatización de la manipulación de las barricas evitará accidente.

- Manipular las pastillas de azufre evitando el contacto directo directo con las mismas, mediante el uso de guantes de protección.

- Señalizar el peligro de asfixia por anhídrido carbónico y hacer un estudio de la ubicación de detectores en las instalaciones y garantizar una buena ventilación de los espacios.

- Conocer las fichas de seguridad los productos químicos manejados como los productos de limpieza. Usar equipos de protección adecuados, como guantes y botas, o bien protecciones respiratorias y oculares.

- El personal debe conocer los riesgos de exposición al metabisulfito de potasio. Se deben usar guantes apropiados, protecciones oculares y respiratorias para evitar que el polvo pueda entrar en contacto con las correspondientes mucosas [10-12].

\subsubsection{Recomendaciones para evitar riesgos asociados al embotellado}

- La estabilidad de las pilas de botellas debe ser asegurada.

- En trabajos que superen los $2 \mathrm{~m}$ de altura, el operario se debe poner cinturón de seguridad.

- Cuando se usa una máquina semi automática, que recoge y suministra las botellas a la línea de embotellado, el operario se sube a una plataforma móvil que no debe superar el metro de altura para despaletizar las camadas altas del pallet. La operación se realizará a pie de suelo para despaletizar las camadas bajas.

- Para evitar golpes y/o cortes, las herramientas cortantes deberán ser usadas con cautela y correctamente y se deberán usar guantes de protección.

- Al retirar las botellas rotas, utilizar guantes apropiados para evitar cortes y usar recogedor y empujador para no tener contacto con la parte cortante.

- Asegurar que las cargas están bien equilibradas y que la horquilla esté bien centrada bajo la carga.

- Se recomienda uso de botas de seguridad con puntera reforzada.

- Usar gafas de seguridad, y ropa que cubra todo el cuerpo para evitar el riesgo de proyecciones de fragmentos. 
- Para labores de limpieza, si se utiliza soplado de aire comprimido, se deberá usar una boquilla de un disco a modo de pantalla de protección y de una regulación de presión que no supere una atmósfera.

- Cerciorarse siempre antes de quitar conexiones, de la inexistencia de presión en las mangueras y del buen estado de conexiones, siempre aflojándolas lentamente.

- Se aconseja, la adecuada señalización para la prohibición de acceso a partes móviles a la zona de la enjuagadora a personal no autorizado.

- Mejorar las posturas en el trabajo para reducir en gran medida los sobreesfuerzos.

- Los accesos a partes móviles de las máquinas deben estar en todo momento protegidos para disminuir los riesgos eléctricos. Las operaciones de mantenimiento deberán realizarse tras haber desconectado el equipo y tomando las medidas necesarias para evitar una puesta en marcha accidental.

- Para disminuir el riesgo de exposición al ruido, se recomienda la colocación de paneles de metacrilato que permitan aislar la maquinaria del resto de la nave.

- Cuando el operario acceda a zonas de riesgo sonoro irá equipado con equipos de protección personal adecuados.

- Se señalizarán las zonas de la bodega donde el uso de protecciones auditivas pueda ser recomendable así como obligatorio. Se realizarán reconocimientos periódicos a los trabajadores expuestos.

- Para disminuir el riesgo derivado del uso de productos químicos, los trabajadores deben conocer los riesgos de exposición a los mismos y las recomendaciones del fabricante para su uso y manipulación.

- Para la manejo del etiquetado se deberán utilizar guantes y mascarillas adecuadas al producto químico que se está aplicando [10,11].

\subsubsection{Recomendaciones para evitar riesgos} asociados al embalaje, paletizado, añejamiento, almacén y expedición

- Para evitar la caída de las botellas, se recomienda realizar la comprobación del correcto funcionamiento del brazo que desplaza las mismas y de los dispositivos de seguridad y no pasar nunca botellas por encima de demás operarios.

- Proteger acceso a partes móviles de las máquinas de la línea y transmisiones en movimiento para evitar golpes y atrapamientos. También la que se encuentren en la zona de zona de despaletizado. Proteger el acceso a dichas zonas bien con protecciones fijas, bien con protecciones móviles asociadas a enclavamiento.

- No dejar pallets en lugares no destinados a ello y mantener siempre limpio y ordenado el lugar de trabajo a fin de disminuir el riesgo de caída.

- Para evitar el sobreesfuerzo, se aconseja realizar pequeñas pausas frecuentemente. También se formará e informará a los trabajadores sobre cuidado postural en el movimiento de cargas.

- A fin de disminuir el riesgo eléctrico se debe realizar la correcta señalización de dicho riesgo en los cuadros eléctricos y mandos, habiendo aleccionado previamente al operario en caso de atasco y sobre actuaciones específicas.

- Usar calzado de seguridad con puntera reforzada y suela antideslizante para evitar posibles accidentes.

- Es esencial el mantenimiento de un adecuado orden y limpieza. Se debe, formar e informar a los operarios de que queda totalmente prohibido colocarse en mecanismos específicos para la carga, ni sobre la parte superior de la misma.

- Los operarios que manipulen las carretillas elevadoras y traspaletas deberán estar instruidos en su manejo y se debe señalizar de la forma efectiva las vías de paso en la bodega $[10,11]$.

\subsubsection{Recomendaciones para evitar riesgos derivados de la realización de actividades auxiliares}

- No usar útiles punzantes y/o cortantes para usos distintos para los cuales han sido destinados.

- Se deberá informar en todo momento de los equipos deteriorados.

- Disponer de cajones con dispositivo de bloqueo que impidan su salida de las guías.

- Es recomendable la instalación de muebles con bordes redondeadas.

- El área de trabajo debe tener una adecuada señalización.

- Colocar y utilizar correctamente las escaleras. Asegurar los elementos de las mismas y colocar apoyos antideslizantes.

- Colocar en zonas de trabajo elevadas, barandillas o barras intermedias.

- Conservar las vías de acceso y los pasos despejados.

- Recubrir el suelo con firme antideslizante evitando irregularidades.

- Hacer pasar los cables junto a las paredes y recoger, cubrir o señalar los que no se puedan colocar así.

- Procurar mantener el orden y limpieza de las infraestructuras.

- Llevar a cabo exámenes periódicos de las instalaciones y equipos eléctricos por personal técnico.

- Comprobar el correcto funcionamiento de los interruptores diferenciales, aislando las partes activas.

- En caso de avería desconectar la tensión, comunicar los daños y hacerlos reparar por personal autorizado.

- Los cuadros eléctricos deberán estar protegidos y señalizados.

- Capacitar a los trabajadores sobre los riesgos de contacto eléctrico directo e indirecto.

- Adquirir equipos de trabajo, teniendo en cuenta el nivel de ruido que produce en circunstancias normales de trabajo. Llevar un adecuado mantenimiento de los mismos.

- Las ventanas orientadas hacia zonas ruidosas deberán contar con doble cristales.

- Las tintas, pegamentos, ozono, y en general, todo producto químico usado también en el laboratorio, deberán estar correctamente etiquetados y contar con sus fichas toxicológicas. Se deberán respetar las indicaciones del fabricante para su manipulación y no se realizarán mezclas de productos que aquel no indique. Al mismo tiempo, el almacenamiento de productos químicos peligrosos se deberá realizar 
en lugares adecuados, en recipientes cerrados y correctamente etiquetados.

- Para evitar el riesgo de incendio, los materiales fácilmente inflamables se deben almacenar en zonas alejadas de fuentes de calor.

- Los aparatos eléctricos se desconectarán cuando no se utilicen.

- Está prohibido fumar.

- No exponer los cartuchos de impresora a temperaturas excesivas o llamas.

- Contar con equipos de extinción de incendios, capacitando a los trabajadores sobre su uso. Realizar mantenimiento periódico de los mismos.

- Señalizar y dejar libres las salidas de emergencia. Realizar simulacros de evacuación contra incendios.

- Para evitar riesgos derivados del uso de equipos para la visualización de datos, utilizar sillas regulables en altura y salvapantallas y establecer pausas breves y frecuentes.

- Dejar espacio suficiente delante del teclado del ordenador para que las manos puedan reposar sobre la mesa y ajustar la altura del teclado a las necesidades del usuario. Disponer de un atril regulable y estable y de reposapiés si fuera necesario. No orientar el puesto de trabajo ni de frente ni de espaldas a la ventana.

- Adecuar la intensidad de la iluminación a la necesidad visual de la tarea.

- Eliminar o apantallar las fuentes de luz deslumbrantes.

- Mantener una correcta limpieza y un mantenimiento periódico de lámparas y luminarias, reparándolas si se encuentran averiadas.

- Impedir reflejos sobre cristales y pantallas de visualización de datos.

- Disponer de una buena instalación de aire acondicionado y calefacción con salidas de aire correctamente orientadas. Evitar corrientes de aire.

- Emplear útiles y mobiliario con diseño ergonómico para evitar posturas forzadas.

- Adecuar el espacio de trabajo a las necesidades del trabajador.

- Facilitar los cambios de postura y pausas en la jornada de trabajo.

- Para evitar accidentes de tráfico durante la realización de tareas de recogida de muestras debe llevarse un mantenimiento adecuado del vehículo, realizar una adecuada planificación del trabajo y el orden del mismo, evitar las prisas y ayudar a que la conducción sea más segura.

- Durante las tareas de laboratorio es fundamental mantener el orden y la limpieza. En caso de derrames se procederá a su limpieza. Además, se deben usar los equipos de protección personal. Está totalmente prohibido comer o fumar en los laboratorios.

- Se debe realizar una revisión periódica de la instalación eléctrica, realizando conexiones seguras, evitando en cualquier caso los empalmes y señalizando los cuadros eléctricos.

- Almacenar los productos comprobando la estabilidad y compatibilidad de los mismos.

- Se dispondrá de un botiquín de primeros auxilios, correctamente señalizado, colgado y cuya situación deberá ser conocida por todos los trabajadores.
- En las tareas de mantenimiento, se debe comprobar el correcto funcionamiento de las paradas de emergencia, finales de carrera y de protecciones de las máquinas.

- Las conexiones a los cuadros eléctricos se realizarán siempre con su correspondiente clavija.

- Los equipos de alumbrado portátiles deben disponer de mango aislante y protección contra golpes.

- Los puntos de atrapamiento como cinta transportadora, bombas, etc., deben estar siempre protegidos.

- En trabajos que superen la altura de $2 \mathrm{~m}$., se debe de llevar puesto un sistema anti caída.

- Para evitar los riesgos de quemadura, se deberá usar protecciones y de manos, así como ropa de trabajo adecuado para labores de soldadura $[10,11]$.

\section{Conclusiones}

La vitivinicultura es una de las actividades económicas más importantes de la Región Cuyo.

En la actualidad existen numerosos procesos productivos, dependiendo de las prácticas enológicas que se lleven a cabo en cada una de las bodegas. A pesar de los múltiples procesos de vinificación, en este trabajo se han considerado todas las posibilidades de producción de vino. En este trabajo, se ha aplicado el concepto de análisis de riesgo a la totalidad del proceso. Se ha hecho hincapié en todas las etapas de este proceso tecnológico y se han identificado los riesgos y peligros potenciales, considerando desde el cultivo de la uva hasta la producción de vino y su almacenamiento y se han planteado medidas que disminuyen y/o eliminen los riesgos y la ocurrencia de accidentes e incidentes.

\section{Referencias}

[1] Instituto Nacional de Vitivinicultura. www.inv. gov.ar

[2] Grainger K., Tattersa H. Producción de vino. Desde la vid hasta la botella. Acribia Editorial. (2007)

[3] Ley Nacional $N^{\circ} 19587$. Higiene y Seguridad en el Trabajo. (1972)

[4] Martín del Moral, A. Manual de buenas prácticas en prevención de riesgos laborales: Sector vitivinícola Ed. UGT La Rioja, Logroño. (2006)

[5] Fundación para la prevención de riesgos laborales.Guía técnica para la implantación de medidas de Seguridad y reglamentación técnica en equipos De trabajo de la actividad agrícola. www . conectapyme . com/documentacion / 2010 Agricola.pdf

[6] Departamento de Salud y Servicios Humanos. Centros para el Control y la Prevención de Enfermedades Instituto Nacional de Salud y Seguridad Ocupacional. Soluciones simples: ergonomía para trabajadores agrícolas. (2002)

[7] Cardona, O. Indicadores de riesgo de desastre y gestión del riesgo. BID. (2005)

[8] Vargas, R. Formulación de un Modelo General para la Gestión del Riesgo en Ciudades. Bogotá. Trabajo de grado (Especialista en evaluación de riesgos y prevención de desastres). Universidad de los Andes. (2002) 
[9] Zolbert, Soto L. Guía de la red para la gestión local del riesgo. Módulos para la capacitación. La red. (1998)

[10] Hiba J., Cicliani A., Cóppola A. Cómo mejorar las condiciones de trabajo y la productividad en empresas agrícolas y agroidustriales. Organización Internacional del Trabajo. (2005)
[11] EN 61346-1. (1998). Industrial systems, installations and equipment and industrial products. Structuring principles and reference designations. Part 1: Basic Rules.

[12] Fisher K. Procedimientos para trabajar en espacios cerrados. Revista Accident Preventión. (1.992) 